\title{
ПРИМЕНЕНИЕ МЕТОДА ТОРSІS ПРИ РЕШЕНИИ ЗАДАЧИ ОЦЕНКИ УСТОЙЧИВОСТИ РАЗВИТИЯ ТЕРРИТОРИЙ *
}

\author{
(C) 2019 Лобкова Елена Валерьевна \\ кандидат экономических наук, доцент кафедры социально-экономического планирования \\ Сибирский федеральный университет, Россия, Красноярск \\ E-mail: elenavalerin@yandex.ru
}

Проведен обзор методических подходов к решению задачи оценки устойчивости социально-экономических систем. Выявлены основные достоинства и недостатки применяемых методик. Описан и апробирован метод TOPSIS как инструмент оценки устойчивости территорий, преодолевающий многие ограничения других подходов. Сформулированы критерии оценки устойчивости региональных социально-экономических систем, обосновано их использование в рамках концепции устойчивости.

Ключевые слова: устойчивое развитие территории, метод TOPSIS, мониторинг, социально-экономические проблемы, инструменты оценки.

Вопросам и проблемам устойчивого развития территорий начиная с 1987 г., когда впервые была принята концепция устойчивого развития, и по настоящее время посвящено большое количество исследований и трудов. В 2015 г. была принята Повестка дня в области устойчивого развития на период до 2030 г., которая содержит 17 целей и 169 задач, направленных на ликвидацию нищеты, сохранение ресурсов планеты и обеспечение благополучия для всего населения. Искоренение нищеты во всех ее формах и проявлениях является одним из необходимых условий устойчивого развития, для обеспечения которого требуется поступательное, всеохватывающее и справедливое экономическое развитие, создание более широких возможностей для всех слоев населения, уменьшение неравенства, повышение основных показателей уровня жизни и качества жизни, поощрение справедливого социального развития, внедрение комплексных и устойчивых методов управления социально-экономическими системами.

Традиционным подходом к выбору инструментов оценки устойчивости является формирование перечня показателей (статистических и экспертных), преобразование их к требуемому для оценки виду и расчет составного индекса устойчивости. Результаты такой процедуры используются для разработки рекомендаций органам управления объекта (территории) и прове- дения сравнительной оценки уровня развития и устойчивости разных стран, регионов, городов и т.д. Обзор авторских подходов и инструментов оценки устойчивости территорий, применяемых в мировой практике, показал, что основная трудность обработки показателей устойчивости заключается в применяемых инструментах для обобщения (агрегирования) данных, приведения их к единой системе измерения, выбор коэффициентов значимости (весомости) показателей и их оценка, а также динамичность среды, в которой исследуемые объекты функционируют, и большой объем значимой для исследования информации.

Вопросы устойчивого развития исследуют авторы С.Н. Бобылев и Б.Н. Порфирьев [2], Т.Н. Гуль [3], А.А. Мальцева [5], О.А. Сидякина, А.В. Саяпин [7], Е.В. Корчагина [4], Е.А. Захарчук, А.Ф. Пасынков [8].

Изучение современных работ авторов в области оценки устойчивости развития территорий показало, что основными подходами и инструментами являются: 1) оценка интегрального показателя устойчивости, который включает ряд параметров социально-экономической устойчивости территории (экономические, производственные, финансово-инвестиционные, бюджетные, социальные и экологические показатели, приведенные к системе индексов); 2) динамический анализ показателей, отражающих

\footnotetext{
* Исследование выполнено в рамках конкурса научных проектов междисциплинарных фундаментальных исследований, проводимого РФФИ совместно с Правительством Красноярского края, в 2018 г. Проект № 18-410242005 р_мк «Разработка методики оценки устойчивого развития территорий Красноярского края».
} 
отдельные аспекты устойчивости, с применением сравнительных характеристик территорий и периодов анализа (экспресс-оценка устойчивости территории на основе мониторинга показателей); 3) расчет коэффициентов и рангов, составление рейтингов анализируемых территорий, основывающихся на статистических и экспертных показателях; 4) методы искусственных нейронных сетей, сценарный подход.

В данном исследовании оценку устойчивого развития территорий предлагается проводить на основе метода TOPSIS (метод упорядоченного предпочтения через сходство с идеальным решением), преимуществом которого является возможность решения задачи принятия решений при бесконечном числе альтернатив. Этапами применения метода TOPSIS являются: построение индексной системы мониторинга, оценки и анализа устойчивости развития территорий; применение метода оценки и метода взвешивания показателей. Модель TOPSIS (The Technique for Order of Preference by Similarity to Ideal Solution) была впервые предложена C.L. Hwang и К. Yoon в 1981 г. [9]. Этот комплексный метод оценки, основанный на расчете дистанции, широко используется для принятия решений отечественными и зарубежными авторами, в основном при оценке надежности транспортных, информационных и инженерных систем [1].

В экономических исследованиях метод используется преимущественно для решения задач оценки конкурентных преимуществ бизнеса и управления персоналом [6].

Модель TOPSIS способна объективно и всесторонне отражать уровень устойчивого развития территории, вычисляя степень близости между оценочной (текущей) ситуацией в социально-экономической системе и ее идеальным состоянием.

В данном исследовании под устойчивым развитием территории понимаем совместное развитие аспектов социально-экономического положения объекта. При оценке уровня устойчивости развития территорий предлагается проводить мониторинг по следующим направлениям:

- производственный критерий оценивает объем производства продукта на территории (в расчете на душу населения) и необходимые для этого элементы производственного потенциала - объем (стоимость) и состояние основных производственных фондов, число предпри- ятий и организаций на территории;

- финансово-инвестиционный аспект устойчивости подразумевает сбалансированное состояние общественных финансов, позволяющее органам власти территории в полной мере исполнять свои обязанности в управляемых сферах; устойчивое финансовое положение организаций и предприятий территории, исполняющих свои обязательства; инвестиционные ресурсы территории, обеспечивающие устойчивость развития производственного сектора;

- социальная составляющая устойчивости развития ориентирована на человека и направлена на улучшение качества жизни в следующих аспектах: здоровья - оценивается через показатель ожидаемой продолжительности жизни; уровня жизни населения - занятость и доходы;

- устойчивость развития региона в аспекте инфраструктуры подразумевает снижение зависимости от транспортных и логистических путей других территорий (регионов), позволяющее сократить издержки производства и улучшить социально-экономическое положение (оценивается плотность дорог, мощность электростанций как необходимое условие производственного процесса, индексы тарифов на грузовые перевозки).

Оценка совместного развития сразу нескольких критериев возможна путем применения метода упорядоченного предпочтения через сходство с идеальным решением (TOPSIS). Множество критериев, которые предполагаются в рамках этого метода, сведем к выделенным направлениям (аспектам) устойчивости. Определяемая альтернатива, согласно концепции TOPSIS, должна иметь самое короткое геометрическое расстояние от положительного идеального решения (PIS) и самое длинное геометрическое расстояние от отрицательного идеального решения (NIS).

В рамках данного метода также встает вопрос определения весов (значимости) используемых критериев (направлений или аспектов устойчивости). Для расчета весовых коэффициентов используются необработанные данные индикаторов и показателей устойчивости в виде матрицы размерностью $\mathrm{m} \bullet \mathrm{n}$, где $\mathrm{m}-$ количество объектов оценки (территорий), $\mathrm{n}$ - количество индикаторов (критериев или показателей устойчивости). Элементы матрицы представим в виде $x_{i j}$ - исходное значение показателя (индикатора) устойчивости. Далее применяется 
стандартная процедура нормализации данных методом линейного масштабирования для приведения значений индикаторов к единым единицам измерения (безразмерным величинам). На следующем этапе для каждого значения показателя проводится процедура стандартизации относительно суммы значений по количеству объектов исследования (территорий):

$$
s_{i j}=\frac{z_{i j}}{\sum_{i=1}^{m} Z_{i j}}
$$

где $s_{i j}$ - стандартизованное значение показателя (индикатора устойчивости); $z_{i j}$ - нормализованное значение показателя (индикатора устойчивости) для конкретного объекта исследования (территории).

Перед завершающей процедурой оценки весов (значимости) индикаторов производится расчет меры энтропии показателя (меры отклонения реального значения от идеального):

$$
\varepsilon_{j}=-\alpha \sum_{i=1}^{m}\left(s_{i j} \ln s_{i j}\right)
$$

где $\varepsilon_{j}$ мера энтропии показателя; коэффициент $\alpha=1 / \ln m$. Весовой коэффициент индикатора устойчивости $\left(\delta_{j}\right)$ определяется по формуле:

$$
\delta_{j}=\frac{\left(1-\varepsilon_{j}\right)}{\sum_{j=1}^{n}\left(1-\varepsilon_{j}\right)}
$$

Далее применяем модель TOPSIS (The Technique for Order of Preference by Similarity to Ideal Solution), которая была впервые предложена C.L. Hwang и К. Yoon в 1981 г. [9]. Модель объективно отражает уровень устойчивого развития территорий путем расчета степени близости между оценочной величиной и ее идеальным решением. В рамках модели строится матрица взвешенных на предыдущем этапе показателей устойчивости. Элементы матрицы находятся по формуле: $\Delta_{i j}=\delta_{j} \bullet z_{i j}$, где $i=1,2, \ldots, m ; j=1,2, \ldots$, $n ; \delta_{j}-$ весовой коэффициент индикатора устойчивости; $z_{i j}$ - нормализованное значение показателя (индикатора устойчивости) для конкретного объекта исследования (территории).

Нахождение лучших оценок показателей $\Delta^{+}$ (положительного идеального решения PIS) и худших оценок показателей $\Delta^{-}$(отрицательного идеального решения NIS) осуществляется следующим образом:

$$
\begin{aligned}
& \Delta^{+}=\left\{\left(\max _{j} \Delta_{i j} \mid j \in J\right), i=1,2, \ldots, m\right\}= \\
& =\left\{\Delta_{1}^{+}, \Delta_{2}^{+}, \ldots \Delta_{n}^{+}\right\}
\end{aligned}
$$

$$
\begin{aligned}
& \Delta^{-}=\left\{\left(\min _{j} \Delta_{i j} \mid j \in J\right), i=1,2, \ldots, m\right\}= \\
& =\left\{\Delta_{1}^{-}, \Delta_{2}^{-}, \ldots \Delta_{n}^{-}\right\}
\end{aligned}
$$

Далее рассчитывается расстояние $S_{i}^{+}$между значением оценки показателя (с учетом его нормализации, стандартизации и взвешивания) и наилучшим (идеальным положительным) значением $\Delta_{j}^{+}$и расстояние $S_{i}^{-}$между значением оценки и наихудшим (идеальным отрицательным) значением $\Delta_{j}^{-}$:

$$
\begin{aligned}
& S_{i}^{+}=\sqrt{\sum_{j=1}^{n}\left(\Delta_{i j}-\Delta_{j}^{+}\right)^{2}} \\
& S_{i}^{-}=\sqrt{\sum_{j=1}^{n}\left(\Delta_{i j}-\Delta_{j}^{-}\right)^{2}}
\end{aligned}
$$

где $i=1,2, \ldots, m$.

Для сопоставления расстояния между оценочным значением показателей и двумя идеальными решениями (положительным и отрицательным) рассчитывается относительный уровень показателя $P_{i}$ :

$$
P_{i}=\frac{S_{i}^{-}}{S_{i}^{+}+S_{i}^{-}}
$$

где $i=1,2, \ldots, m$.

Оценка устойчивого развития территорий согласно данному подходу производится по модели TOPSIS и включает несколько направлений (критериев): производственный аспект $(\Pi)$; социальный аспект $(C)$; финансово-инвестиционный (ФИ); инфраструктурный аспект (И). Итоговое значение совокупного индикатора устойчивости территории, учитывающего весовые коэффициенты $w_{i}$, определяется следующим образом: $P_{i(S T)}=P_{i(\Pi)} \bullet \delta_{i(\Pi)}+P_{i(C)} \bullet \delta_{i(C)}$ $+P_{i(Ф И)} \bullet \delta_{i(Ф И)}+P_{i(И)} \bullet \delta_{i(И)}$, где $i=1,2, \ldots, m$; $P_{i(S T)}-$ совокупный индикатор устойчивости $i-$ й территории; $P_{i(\Pi)}, P_{i(C)}, P_{i(Ф И)}, P_{i(И)}-$ оценочные значения производственной, социальной, финансово-инвестиционной и инфраструктурной устойчивости; $\delta_{i(\Pi)}, \delta_{i(C)}, \delta_{i(Ф И)}, \delta_{i(И)}-$ весовые значения производственного, социального, финансово-инвестиционного и инфраструктурного аспектов устойчивости.

Предложенный инструментальный подход был апробирован в процессе решения задачи оценки устойчивости территории. Такого типа 
задачи осложнены всегда выбором показателей и оценкой уровня значимости отобранных критериев. Весовые коэффициенты или коэффициенты относительности важности критериев и показателей часто оцениваются экспертно, что делает авторский подход субъективным и снижает степень доверия к результатам оценки.
В таблице 1 приведены отобранные в данном исследовании показатели устойчивости и их группировка по критериям, а также оцененные на основании расчета меры энтропии показателя (меры отклонения реального значения от идеального) коэффициенты относительной важности показателей и критериев.

Таблиц̧а 1. Критерии и показатели устойчивости, коэффициенты относительной важности $(\delta)$

\begin{tabular}{|c|c|c|c|}
\hline Критерии & $\delta$ критериев & Частные показатели устойчивости & $\delta_{j}$ \\
\hline \multirow{4}{*}{$\begin{array}{l}\text { Производственный } \\
P_{i(\Pi)}\end{array}$} & \multirow{4}{*}{0,14629} & Валовой региональный продукт на душу населения, руб. & 0,03759 \\
\hline & & Степень износа основных фондов,\% & 0,01718 \\
\hline & & Стоимость основных фондов, млн. руб. & 0,04398 \\
\hline & & Число предприятий и организаций & 0,04755 \\
\hline \multirow{4}{*}{ Социальный $P_{i(C)}$} & \multirow{4}{*}{0,15101} & Ожидаемая продолжительность жизни при рождении, лет & 0,04207 \\
\hline & & $\begin{array}{l}\text { Доля населения с денежными доходами выше величины } \\
\text { прожиточного минимума, установленной в субъекте Рос- } \\
\text { сийской Федерации,\% }\end{array}$ & 0,01093 \\
\hline & & $\begin{array}{l}\text { Уровень занятости, в\% к численности населения в возрасте } \\
15-72 \text { лет }\end{array}$ & 0,01257 \\
\hline & & Среднедушевые денежные доходы населения, руб. в мес. & 0,08543 \\
\hline \multirow{4}{*}{$\begin{array}{l}\text { Финансово- } \\
\text { инвестиционный } \\
P_{i(Ф И)}\end{array}$} & \multirow{4}{*}{0,12800} & $\begin{array}{l}\text { Доходы минус расходы (сальдо) консолидированных бюд- } \\
\text { жетов субъектов Российской Федерации, млн. руб. }\end{array}$ & 0,05019 \\
\hline & & $\begin{array}{l}\text { Удельный вес убыточных организаций, в\% от общего числа } \\
\text { организаций }\end{array}$ & 0,02067 \\
\hline & & $\begin{array}{l}\text { Просроченная задолженность по заработной плате в расче- } \\
\text { те на одного работника, } \\
\text { перед которым имеется просроченная задолженность, руб. }\end{array}$ & 0,01765 \\
\hline & & Инвестиции в основной капитал на душу населения, руб. & 0,03948 \\
\hline \multirow{4}{*}{$\begin{array}{l}\text { Инфраструктурный } \\
P_{i(И)}\end{array}$} & \multirow{4}{*}{0,24410} & $\begin{array}{l}\text { Плотность железнодорожных путей, км путей на } 10000 \text { км² } \\
\text { территории }\end{array}$ & 0,05665 \\
\hline & & $\begin{array}{l}\text { Плотность автомобильных дорог общего пользования с } \\
\text { твердым покрытием, км путей на } 1000 \text { км² территории }\end{array}$ & 0,06329 \\
\hline & & Мощность электростанций, млн. кВт & 0,07631 \\
\hline & & Индексы тарифов на грузовые перевозки,\% & 0,04784 \\
\hline
\end{tabular}

Таблица 2. Значения критериев устойчивости регионов и относительный уровень устойчивости территории

\begin{tabular}{|l|c|c|c|c|c|}
\hline \multicolumn{1}{|c|}{ Регион } & $P_{i(\Pi)}$ & $P_{i(C)}$ & $P_{i(\Phi И)}$ & $P_{i(И)}$ & $\begin{array}{c}\text { Сводное зна- } \\
\text { чение } P_{i(S T)}\end{array}$ \\
\hline Красноярский край & 0,79662 & 0,78322 & 0,72627 & 0,81031 & 0,52557 \\
\hline Новосибирская область & 0,78182 & 0,74154 & 0,73134 & 0,81803 & 0,51965 \\
\hline Иркутская область & 0,75143 & 0,82890 & 0,70242 & 0,72914 & 0,50299 \\
\hline Алтайский край & 0,69595 & 0,63486 & 0,64204 & 0,75351 & 0,46380 \\
\hline Кемеровская область & 0,67357 & 0,53751 & 0,71712 & 0,77695 & 0,46115 \\
\hline Республика Хакасия & 0,67045 & 0,67393 & 0,52051 & 0,69455 & 0,43602 \\
\hline Забайкальский край & 0,62241 & 0,69053 & 0,59755 & 0,61946 & 0,42303 \\
\hline Омская область & 0,63217 & 0,54779 & 0,55747 & 0,71405 & 0,42086 \\
\hline Республика Алтай & 0,61121 & 0,75084 & 0,57422 & 0,55415 & 0,41157 \\
\hline Томская область & 0,58648 & 0,59358 & 0,43273 & 0,62015 & 0,38220 \\
\hline Республика Бурятия & 0,46336 & 0,29208 & 0,52004 & 0,60164 & 0,32532 \\
\hline Республика Тыва & 0,38751 & 0,59611 & 0,39271 & 0,25576 & 0,25941 \\
\hline
\end{tabular}


Расчеты были произведены на примере регионов Сибирского федерального округа по данным Росстата за 2017 г. При отборе показателей и формировании критериев устойчивости во внимание принимался подход к устойчивости территории как явлению совместного развития всех выделенных аспектов социально-экономического положения субъекта.

В основе количественной оценки устойчивости развития территорий традиционно лежит набор измеряемых показателей и индикаторов для проведения регулярного мониторинга ситуации. Полученные результаты оценки требуют уточнения и анализа полученного рейтинга территорий Сибирского федерального округа. Автором был показан пример применения метода TOPSIS для решения задачи оценки комплексного показателя, включающего несколько критериев и индикаторов, с разной степенью важности.

\section{Библиографический список}

1. Антамошкин О.А. Система поддержки принятия решений на основе многоатрибутивных методов // Вестник СибГАУ. 2009. 4(25). С. 69-71.

2. Бобылев С. Н., Порфирьев Б.Н. Устойчивое развитие крупнейших городов и мегаполисов: фактор экосистемных услуг // Вестник Московского университета. 2016. № 6. С. 3-21.

3. Гуль Т.Н. Оценка устойчивости развития региона // Социально-экономические явления и процессы. 2011. № 10(032). С. 34-39.

4. Корчагина E.B. Методы оценки устойчивого развития региональных социально-экономических систем // Проблемы современной экономики. 2012. № 1. С. 67-71.

5. Мальщева А.А. Методические подходы к оценке устойчивости территорий инновационного развития с использованием теории динамических нормативов // Международный бухгалтерский учет. 2016. № 6 (396). С. 41-60.

6. Мамедова М.Г., Джабраилова З.Г. Многокритериальная оптимизация задач управления человеческими ресурсами на базе модифицированного метода TOPSIS // Восточно-Европейский журнал передовых технологий. 2015. 2/4 (74). С. 48-61.

7. Сидякина О.А., Саялин А.В. Инструментарий обеспечения устойчивого развития агропромышленного региона // Социально-экономические явления и процессы. 2015. № 4. С. 81-89.

8. Захарчук Е.А., Пасынков А.Ф. Концептуальные основы формирования финансовой устойчивости территории: теоретико-методологический подход // Экономика региона. 2008. № 4. С. 108-115.

9. Hwang, C. L., Yoon K. Multiple attributes decision making methods and applications. Berlin, Springer-Verlag Berlin Heidelberg. 1981.269 p. DOI: 10.1007/978-3-642-48318-9. 